\title{
Les actions de l'ESRF destinées au public et aux scolaires
}

Dominique Cornuéjols (dominique.cornuejols@esrffr)

Chargée de communication, ESRF, BP 220, 38043 Grenoble Cedex 9

En tant que centre de recherche européen financé par dix-neuf pays, I'ESRF

se doit de communiquer largement sur son activité et ses résultats. Les moyens de communication sont très variés, allant du site internet aux communiqués de presse envoyés aux médias du monde entier, l'accueil de visiteurs, l'événementiel ou des actions spécifiques destinées aux scolaires.

Pour renforcer l'efficacité de ses activités de communication, l'ESRF a développé de nombreux partenariats, aussi bien internationaux que locaux.

\section{Site internet et médias}

Le site www.esrf.eu est le moyen privilégié pour diffuser les informations scientifiques, technologiques ou événementielles de l'ESRF, aussi bien vers la communauté des utilisateurs de la lumière synchrotron que vers le public. Un grand soin est apporté à la rédaction des articles. Nous développons depuis quelques années notre présence dans les médias sociaux comme Twitter. L'ESRF participe également au site collaboratif www.lightsources.org, qui réunit les synchrotrons et lasers à électrons libres. Du côté vidéo, la chaîne YouTube lightforscience a été regardée jusqu'à maintenant par près de 100000 internautes.

L'ESRF est en relation avec les grands médias des pays membres et communique régulièrement pour toucher le public. On peut noter ici que la communication avec ces médias n'est pas toujours facile. En effet, les synchrotrons, où la science et la technologie sont très transversales, ne sont pas identifiés aussi facilement qu'un collisionneur de particules ou qu'un laboratoire de biologie. Les thématiques reprises par les grands médias tournent souvent autour du médical, du patrimoine culturel ou de la paléontologie, et ne sont pas toujours représentatives de toute l'activité d'un synchrotron, malgré nos efforts pour élargir les champs d'intérêt du public.

\section{Les visites}

L'ESRF a depuis toujours accordé une grande importance aux visites de groupes (étudiants, professionnels de tous horizons, scolaires et grand public) et reçoit chaque année environ 3500 visiteurs, dont la moitié viennent de l'étranger.
Des jeunes scientifiques, thésards ou postdocs, sont formés régulièrement à l'accompagnement des groupes et aux problématiques "science et société ". L'immersion des groupes dans le monde de la recherche et le contact direct avec les scientifiques sont la force de ces visites, qui ont beaucoup de succès. L'ESRF a mis sur pied un partenariat avec l'Office de Tourisme de Grenoble, offrant ainsi à chacun la possibilité de découvrir l'environnement remarquable d'un synchrotron. Nous recevons également de nombreuses classes du secondaire, pour lesquelles nous adaptons le niveau du discours scientifique. Des groupes scolaires viennent fréquemment des pays voisins, et nous organisons dans ce cas la visite dans leur langue.

Les visites, dont la qualité s'est renforcée au cours des dernières années, reposent largement sur un ensemble d'outils de communication développés spécifiquement pour accueillir les groupes dans les meilleures conditions : maquettes, brochures, posters, vidéos et vitrines de démonstration. Le site internet de l'ESRF mettra prochainement à la disposition des professeurs et des élèves de nouvelles ressources pédagogiques. Une version française de ces pages est envisagée.

L'ESRF investit actuellement dans l'équipement d'un nouvel espace visiteurs, d'une surface de $200 \mathrm{~m}^{2}$, surplombant le nouveau hall d'expériences par une grande baie vitrée. Cet espace, à la scénographie très sophistiquée, permettra aussi de recevoir des groupes scolaires autour d'ateliers sur la découverte de la matière et de la lumière sous forme de petites expériences "hands-on" (apprentissage pratique). 


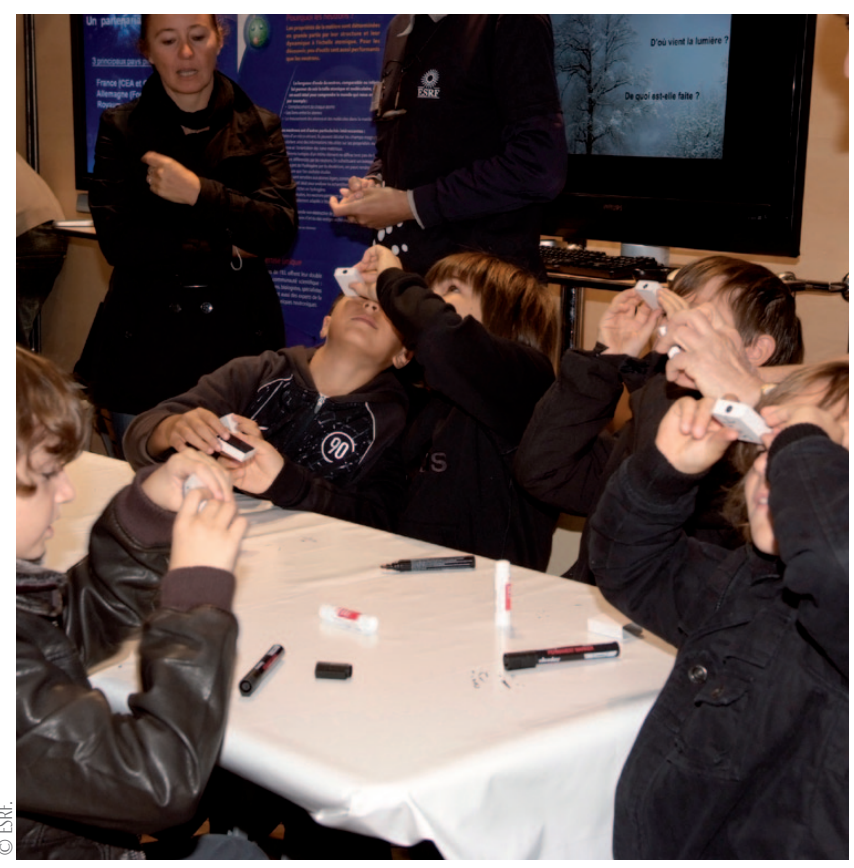

Des enfants fabriquent et testent leur propre spectroscope sur le stand ESRF à la Fête de la Science.

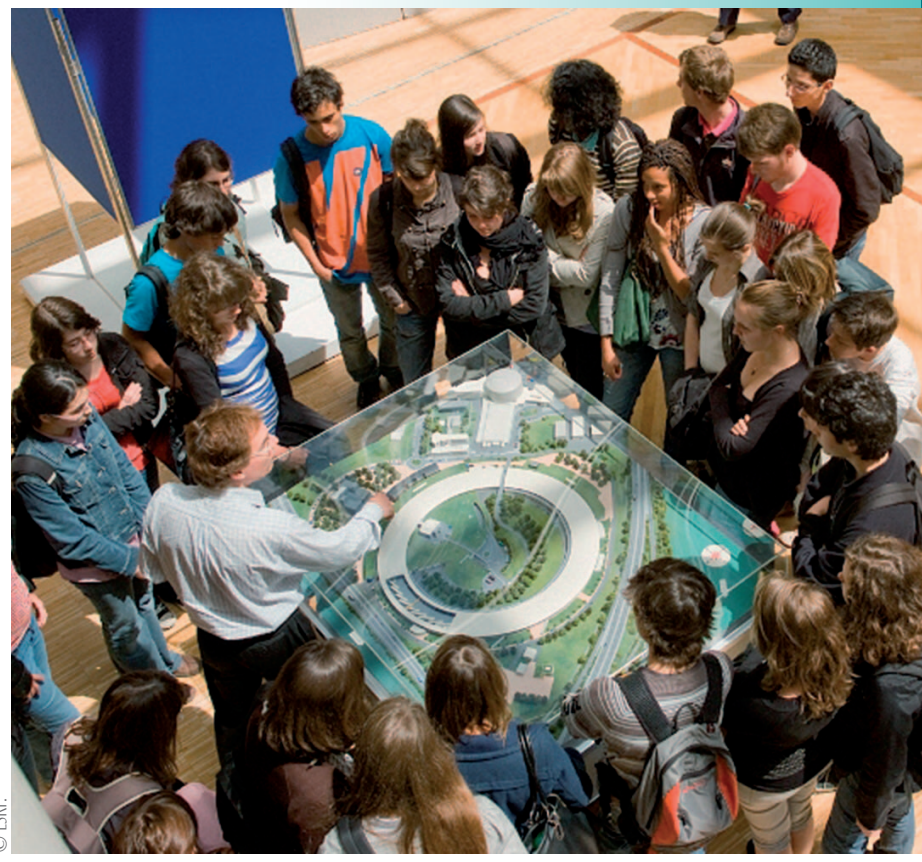

Groupe de jeunes visiteurs autour d'une maquette de l'ESRF.

\section{Les événements}

\section{(portes ouvertes, Fête de la Science...)}

L'ESRF accueille régulièrement le public à l'occasion d'événements « portes ouvertes » et participe chaque année à la Fête de la Science en Isère. Ces activités sont, la plupart du temps, organisées en collaboration avec d'autres grandes infrastructures de recherche européennes du site, l'ILL (Institut Laue-Langevin) et l'EMBL (Laboratoire européen de biologie moléculaire), mais aussi plus largement dans le cadre du campus d'innovation GIANT $^{(1)}$, dont l'ESRF fait partie.

Un collectif de scientifiques s'est formé à Grenoble il y a un an autour des " 100 ans de cristallographie », afin de mettre en valeur cette science méconnue du public et pourtant d'une grande actualité, pour laquelle plusieurs prix Nobel ont été décernés ces dernières années. Réunissant des scientifiques de GIANT, de l'Université de Grenoble, du CEA, du CNRS, ainsi que de l'ESRF, de l'ILL et de l'EMBL, ce collectif a en vue l'Année internationale de la cristallographie en 2014 et la réalisation d'événements à grande échelle. Il travaille aussi en étroite collaboration avec le CCSTI (Centre de culture scientifique technique et industrielle) de Grenoble. Pour en savoir plus sur les actions passées et à venir :

www.echosciences-grenoble.fr/ sites/100-ans-de-cristallographie
Parmi les activités en cours les plus originales, on peut citer les projets "Art et Science ». L'ESRF, dans le cadre du projet européen ISWA (Immersion in the science worlds through arts), a reçu plusieurs jeunes artistes en résidence dans ses locaux. Ces artistes ont produit des œuvres d'art qui ont été diffusées en Europe pour inspirer les jeunes (14-20 ans) et les inciter à créer leurs propres œuvres.

Un autre projet, Thinkrotron, imaginé par le plasticien Laurent Mulot, a vu le jour récemment. Il s'agit de mettre en scène les relations entre les citoyens, la recherche et les scientifiques. Ce projet, également en collaboration avec le CCSTI-La Casemate de Grenoble, sera présenté sous la forme d'une exposition à la prochaine Biennale Art-Science de Grenoble, "les rencontres imaginaires ", du 3 au 13 octobre $2013^{(2)}$.

\section{"Science in School", une initiative européenne}

Avec ses sept partenaires d'EIROforum ${ }^{(3)}$, l'ESRF est coéditeur de la revue Science in School à destination des professeurs du secondaire en Europe, mais en réalité diffusée dans le monde entier. La démarche éditoriale est unique : on trouve dans cette revue des articles écrits par des scientifiques pour des professeurs, mais aussi des articles écrits par des professeurs pour leurs pairs, afin de partager leurs expériences pédagogiques autour de l'apprentissage des sciences et techniques.
Cette revue est diffusée sous forme papier, mais également sur internet, avec des traductions multilingues reposant sur le bénévolat des professeurs :

www.scienceinschool.org

Dans le même état d'esprit, l'ESRF, l'ILL et l'EMBL ont organisé des "stages en immersion » pour les professeurs du secondaire d'une vingtaine de pays d'Europe (EIROforum Teachers School). Les professeurs découvrent à cette occasion les laboratoires, les instruments de l'ILL et les lignes de lumière de l'ESRF, mais échangent également entre eux pour construire des activités pédagogiques autour des sources de neutrons et de rayons $\mathrm{X}$.

(1) GIANT (Grenoble innovation for advanced new technologies), campus d'innovation, regroupe des partenaires de la recherche, de l'enseignement supérieur et de l'industrie dans les trois grands domaines suivants

- les technologies de l'information et de la communication,

- les énergies renouvelables et les questions environnementales,

- les biosciences et la santé.

(2) Dans cette rencontre avec le public, qui se tiendra au muséum de Grenoble, Laurent Mulot infiltrera la mémoire informatique du synchrotron ESRF et y installera un invité-surprise : la poésie. http://rencontre-i.eu/exposition

(3) EIROforum est un partenariat entre huit organisations de recherche intergouvernementales CERN, EFDA-JET, EMBL, ESA, ESO, ESRF, European XFEL et ILL. 